\title{
Protective effect of neutralizing anti-IL-18 $\alpha$ monoclonal antibody on a mouse model of acute graft-versus-host disease
}

\author{
XIAOCUI LI ${ }^{1 *}$, CUIPING ZHANG ${ }^{1,3^{*}}$, WEI CHEN ${ }^{2}$, BIN PAN $^{2}$, FANYUN KONG ${ }^{1}$, \\ KUIYANG ZHENG ${ }^{1}$, RENXIAN TANG ${ }^{1}$ and LINGYU ZENG ${ }^{2}$
}

\author{
${ }^{1}$ Department of Pathogenic Biology and Immunology, Laboratory of Infection and Immunity, Xuzhou Medical College, \\ Xuzhou, Jiangsu 221004; ${ }^{2}$ Blood Diseases Institute, Xuzhou Medical College; Key Laboratory of Bone Marrow Stem Cells; \\ Department of Hematology, The Affiliated Hospital of Xuzhou Medical College, Xuzhou, Jiangsu 221002; \\ ${ }^{3}$ Clinical Laboratory of The TCM Hospital of Huaian, Huaian, Jiangsu 223001, P.R. China
}

Received April 7, 2015; Accepted June 24, 2015

DOI: $10.3892 /$ or.2015.4176

\begin{abstract}
Graft-versus-host disease (GVHD) is a devastating complication of hematopoietic stem cell transplantation (HSCT), and is characterized by systemic inflammation and tissue damage in multiple organs, such as the liver and small intestine. Interleukin-18 (IL-18), an important pro-inflammatory cytokine, is elevated during the course of acute GVHD (aGVHD), and is associated with the severe clinical manifestations of the disease. The biological activity of IL-18 is based on its interaction with the IL-18 receptor (IL-18R) expressed in a variety of cells. The aim of this study was to assess whether blocking the interaction of IL-18 with IL-18R by the antiIL-18R $\alpha$ antibody could attenuate the severity of aGVHD. We used a well-established mouse bone marrow transplantation $(\mathrm{BMT})$ model $(\mathrm{B} 6 \rightarrow \mathrm{BALB} / \mathrm{c})$ to block the IL-18/IL-18R interaction by a neutralizing monoclonal antibody $(\mathrm{mAb})$ against murine IL-18R $\alpha$. Administration of anti-IL-18R $\alpha \mathrm{mAb}$ had a significant protective effect on the clinical and pathologic manifestations of aGVHD, resulting in a markedly improved survival rate, modified inflammatory response and decreased tissue damage. Interfering with IL-18/IL-18R interaction affected levels of Th1, Th2 and Th17 subsets in the peripheral blood of the aGVHD animals. Additionally, it led to decreased tissue expression of IL-18 and apoptosis-associated molecules (Fas and FasL), and lower phosphorylation levels of p38MAPK
\end{abstract}

Correspondence to: Dr Lingyu Zeng, Blood Diseases Institute, Xuzhou Medical College, Xuzhou, Jiangsu 221002, P.R. China E-mail: zengly2000@163.com

Dr Renxian Tang, Department of Pathogenic Biology and Immunology, Laboratory of Infection and Immunity, Xuzhou Medical College, Xuzhou, Jiangsu 221004, P.R. China

E-mail: tangrenxian-t@163.com

${ }^{*}$ Contributed equally

Key words: acute graft-versus-host disease, IL-18R $\alpha$, apoptosis, Fas, p38MAPK in the liver and small intestine. These changes coincided with the decrease in cell apoptosis in aGVHD target organs. Thus, anti-IL-18R $\alpha$ therapy may, therefore, represent a new therapeutic interference approach for treating aGVHD.

\section{Introduction}

Hematopoietic stem cell transplantation (HSCT) is a curative therapy for a variety of diseases, especially for hematological malignancies and immunodeficiency diseases. However, graft-versus-host disease (GVHD) is still a major cause of post-transplant mortality among patients receiving $\operatorname{HSCT}(1,2)$. Based on the time frame and type of organ involvement, GVHD can be characterized as acute (aGVHD) and chronic (cGVHD). Despite recent advances in therapies to improve the outcome of aGVHD in patients, this complication still occurs in $30-50 \%$ of all HSCT recipients $(2,3)$. The pathophysiology of aGVHD is characterized by the enhanced recognition of host alloantigens by alloreactive donor $\mathrm{T}$ cells, proliferation, differentiation and secretion of cytokines, host cell apoptosis and tissue damage (4-6). Studies show an association of both donor $\mathrm{CD}^{+}$and $\mathrm{CD}^{+} \mathrm{T}$ cells with aGVHD (4). After activation by host alloantigens, $\mathrm{CD} 4^{+} \mathrm{T}$ cells differentiate into various $\mathrm{T}$ helper (Th) cell subsets, such as Th1 and Th17 cells that are often increased in aGVHD and play a role in mediating aGVHD-induced tissue damage (7), and Th2 cells that apparently have the protective role in the development of aGVHD (8). In contrast to $\mathrm{CD}^{+} \mathrm{T}$ cells, activated $\mathrm{CD} 8^{+}$ $\mathrm{T}$ cells mainly differentiate into cytotoxic $\mathrm{T}$ lymphocytes (CTLs), and the damage induced by activated $\mathrm{CD}^{+} \mathrm{T}$ cells in aGVHD primarily depends on the cytolytic machinery (9-11). Although the role of different $T$ cell subsets in aGVHD has been investigated, the key molecular mechanisms that trigger and maintain abnormality of these $\mathrm{T}$ cell subsets remain unclear.

Interleukin-18 (IL-18) is a member of the IL-1 cytokine superfamily and is produced by a variety of cells. The receptor for IL-18 (IL-18R) consists of a ligand-binding (IL-18R $\alpha$ ) and a signal-transducing chain (IL-18R $\beta$ ), both essential for signal transduction (12). Increased IL-18 serum levels were reported in patients with aGVHD, and further studies have shown that 
IL-18 stimulates the Th1 cell-mediated immune response, enhances expansion and cytotoxic activity of donor CD8 $8^{+}$ $\mathrm{T}$ cells, and increases pro-inflammatory cytokine secretion in the course of aGVHD $(10,13)$. In the present study, we evaluated the protective effect of IL-18R neutralizing antibody $(\mathrm{Ab})$ in an experimental aGVHD model. We analyzed the changes in the clinical manifestations of aGVHD, Th cell subsets, systemic inflammation and cell apoptosis that resulted from anti-IL-18R $\alpha$ monoclonal antibody (mAb) administration. We suggest that blocking the interaction of IL-18 with IL-18R may be beneficial for the treatment of aGVHD.

\section{Materials and methods}

Animals. The mice were purchased from the Experimental Animal Center of Yangzhou University (Yangzhou, China). Six- to 8-week-old female donor C57BL/6 (B6, H-2 $)$ mice and 8- to 10-week-old recipient BALB/c $\left(\mathrm{H}-2^{\mathrm{d}}\right)$ mice were caged in a pathogen-free controlled environment with a 12-h light/dark cycle. Animals were fed and provided water ad libitum for $\sim 2$ weeks. All animal experiments were approved by the Animal Ethics Committee of Yangzhou University.

Induction of a GVHD and immunologic interventions. aGVHD was induced in mice as previously described (14). Briefly, the transplantation day was set as day 0 . On day 0 , donor C57BL/6 mice were sacrificed by cervical dislocation, and the femur, tibia and spleen were harvested and kept in ice-cold phosphate-buffered solution (PBS). Bone marrow cells (BMCs) were flushed from the femur and tibia, and a single-cell BMC suspension in PBS was prepared. Donor spleen cells (SPs) were minced and a single-cell suspension was obtained by passage of minced spleen through a $75-\mu \mathrm{m}$ wire mesh strainer and resuspended in RPMI-1640 medium (Life Technologies, Carlsbad, CA, USA). Recipient BALB/c mice were divided into 2 groups. Mice in the normal control group $(n=6)$ received no treatment, while the rest of the animals $(n=12)$ received $7.5 \mathrm{~Gy}$ total body irradiation from a Softex M-150 WE ${ }^{60} \mathrm{Co}$ source (Softex, Tokyo, Japan). After a 4-h irradiation, the mice were randomly divided into 2 experimental groups, $\mathrm{BS}+\mathrm{Ab}$ and BS (6 animals/group), and were injected intravenously with $0.25 \mathrm{ml}$ RPMI- 1640 medium containing $5 \times 10^{6}$ donor BMCs combined with $5 \times 10^{5}$ donor SPs. Recipient mice in the $\mathrm{BS}+\mathrm{Ab}$ group also received $10 \mu \mathrm{g} /$ mouse intraperitoneal injection of neutralizing $\mathrm{mAb}$ against murine IL-18R $\alpha$ (catalog no. MAB12161; R\&D Systems, Minneapolis, MN, USA) every 2 days. Recipient mice in the BS group received an intraperitoneal injection of PBS.

Assessment of aGVHD. Recipient mice were monitored daily for the following clinical manifestations of aGVHD: weight loss, hunched posture, poor activity, ruffled fur and loss of skin integrity $(14,15)$. The severity of the above symptoms was scored from 0 to 2 . The sum of the scores for all the symptoms for each mouse (maximum 10) was used as an index of severity and progression of aGVHD. Each experiment was repeated 4-5 times independently.

Flow cytometric analysis. Blood samples were collected from the animals in all groups weekly starting from day 7 and up to day 35 post-transplantation (P.T.). Th1, Th17 and Th2 in peripheral blood were detected by intracellular cytokine staining as previously described (16). Briefly, blood samples were stimulated with $50 \mathrm{ng} / \mathrm{ml}$ phorbol myristate acetate (PMA) and $750 \mathrm{ng} / \mathrm{ml}$ ionomycin (both from Sigma, St. Louis, MO, USA) in the presence of $10 \mu \mathrm{g} / \mathrm{ml}$ brefeldin A (Life Technologies) at $37^{\circ} \mathrm{C}$ for $4 \mathrm{~h}$. The cells were then harvested and surface staining was performed for 15-20 min with a mixture of the FITC-CD4 and PE-CD45 antibodies. After being washed with PBS, the cells were fixed and penetrated with Fix and Perm buffer (An Der Grub, Austria), followed by staining with Per-CP-conjugated IFN- $\gamma$, IL-17A, IL-4, or IL-6 monoclonal antibodies. All antibodies were purchased from BD Biosciences (San Jose, CA, USA). All data were acquired using FACSCalibur flow cytometer and analyzed with CellQuest software (both from BD Biosciences).

Cytometric bead array and enzyme-linked immunosorbent assay (ELISA). The levels of serum cytokines were determined weekly during a time course of 5 weeks (week 1 to 5 P.T.) using the Cytometric Bead Array ${ }^{\mathrm{TM}}$ Mouse Th1/Th2/Th17 Cytokine kit (BD Biosciences). Mouse cytokine-specific bead sets and standards were implemented according to the manufacturer's instructions. The fluorescence produced by the beads was measured using FACSCalibur flow cytometer and analyzed with the accompanying software.

Serum levels of IL-18 were assessed by ELISA. For each sample, $0.1 \mathrm{ml}$ of supernatants and standards was assayed in duplicates using a mouse IL-18 immunoassay kit from BlueGene Systems (Shanghai, China) according to the manufacturer's instructions.

Real-time PCR analysis. Total RNA was extracted using TRIzol (Life Technologies) and cDNA was synthesized from $1 \mu \mathrm{g}$ of RNA using miRcute miRNA cDNA Synthesis kit (Tiangen, Beijing, China). The PCR primers for the target genes were as follows: IL-18, 5'-tgacaacacgtttactttatacct-3' (sense) and 5'-cacagccagtcctctacttca-3' (antisense); Fas, 5'-agttt catgaacccgectc-3' (sense) and 5'-gcagacatgctgtggatctg-3' (antisense); FasL, 5'-ttaaatgggccacactcctc-3' (sense) and 5'-actccgtga gttcaccaacc-3' (antisense); and $\beta$-actin, $5^{\prime}$-atggaggggaatacag ccc-3' (sense) and 5'-ttctttgcagctccttcgtt-3' (antisense). mRNA expression levels of the target genes were analyzed by real-time qPCR using Fast SYBR-Green Master Mix and an Applied Biosystems ${ }^{\circledR}$ instrument (both from Life Technologies). qPCR reaction was carried out as follows: denaturation for $2 \mathrm{~min}$ at $94^{\circ} \mathrm{C}$, followed by 40 cycles of $30 \mathrm{sec}$ at $94^{\circ} \mathrm{C}, 30 \mathrm{sec}$ at $60^{\circ} \mathrm{C}$ and $1 \mathrm{~min}$ at $2^{\circ} \mathrm{C}$. The baseline adjustment method of the HRM software was used to determine the $\mathrm{Ct}$ in each reaction. All samples were amplified in duplicates, and the mean was used for further analysis. Gene expression was normalized to $\beta$-actin.

Western blotting. Mice were sacrificed after bone marrow transplantation (BMT) at indicated times, and the liver and small intestine were resected aseptically. Partial tissues were flash-frozen in liquid nitrogen immediately upon dissection, while the rest of the tissues were fixed with $4 \%$ paraformaldehyde solution for further analysis as described below. Individual frozen samples of liver and small intestine 
were homogenized with an electric hand-held precise tissue homogenizer (PRO200; Pro Scientific, USA) in a 5X volume of ice-cold homogenization buffer (Kangchen, Shanghai, China). Protein concentrations were quantified using a BCA protein assay (Thermo Scientific, Waltham, MA, USA). Equal amounts of proteins were separated on $12 \%$ SDS-PAGE and transferred to PVDF membranes (Millipore, Bedford, MA, USA). Membranes were blocked with 5\% non-fat milk in Trisbuffered saline (TBS) for $3 \mathrm{~h}$ at room temperature and then incubated with p38MAPK and phospho-38MAPK antibodies (Cell Signaling Technology, Danvers, MA, USA) overnight at $4^{\circ} \mathrm{C}$ followed by incubation with HRP-conjugated anti-rabbit or anti-mouse IgG (Sigma). Detection was performed with the enhanced chemiluminescence reagent (Millipore). GAPDH was used as a loading control. Densitometry was performed to compare protein expression among the 3 groups using Bio-Rad Quantity One software (Bio-Rad, Hercules, CA, USA).

Histopathology and immunohistochemical analysis. Liver and small intestine samples were fixed with $4 \%$ paraformaldehyde solution as described above, ethanol-dehydrated and embedded in paraffin. Tissues were then sectioned and stained with hematoxylin and eosin (H\&E). Scoring was performed as previously described ( 0 as normal, 0.5 as focal and rare, 1.0 as focal and mild, 2.0 as diffuse and mild, 3.0 as diffuse and moderate, and 4.0 as diffuse and severe). Histological examination and lesion assessment were independently carried out by two pathologists in a blinded fashion.

Immunohistochemistry (IHC) was performed using rabbit polyclonal IL-18, Fas and FasL antibodies (Abcam, Cambridge, UK), respectively. Briefly, 4- to 5- $\mu \mathrm{m}$ sections were deparaffinized, and antigen retrieval was performed in citrate buffer using a microwave oven. Slides were rinsed, treated with $3 \% \mathrm{H}_{2} \mathrm{O}_{2}$ in methanol and blocked by goat serum (Sigma) in Tris-buffer. Slides were incubated overnight at $4^{\circ} \mathrm{C}$ with a primary antibody diluted in blocking solution. After rinsing in Tris-buffer, the slides were incubated with biotin-labeled secondary anti-rabbit IgG (Abcam). The sections were then incubated with the ABC-HRP complex (ZSGB-Bio, Beijing, China). Binding sites were visualized with diaminobenzidine/hydrogen peroxide, followed by counterstaining with hematoxylin.

TUNEL assay. The apoptotic cells in target organs were detected using the terminal deoxynucleotidyl transferase-mediated deoxyuridine triphosphate nick-end labeling (TUNEL) assay (Roche, Basel, Switzerland). All target tissues were fixed in freshly prepared $4 \%$ paraformaldehyde solution. Tissue sections $(4-\mu \mathrm{m})$ were prepared on glass slides. TUNEL assay was performed according to the manufacturer's instructions. Briefly, sections were deparaffinized in xylene, dehydrated in ethanol, and incubated with $20 \mu \mathrm{g} / \mathrm{ml}$ proteinase $\mathrm{K}$ for $30 \mathrm{~min}$ at room temperature. Sections were then incubated with TUNEL reaction mixture for $60 \mathrm{~min}$ at $37^{\circ} \mathrm{C}$ in a humidified atmosphere in the dark and analyzed under a Olympus BX50 fluorescence microscope (Olympus, Japan).

Three tissue slices were randomly selected from each group, and five high-power fields were randomly selected from each slice. The number of apoptotic and normal cells were counted. The apoptosis index (AI) for each target organ was calculated using the following equation: $\mathrm{AI}(\%)=($ the number of apoptotic cells/number of normal cells) x $100 \%$.

Statistical analysis. Experimental data are expressed as means \pm SD. Differences between two groups were analyzed by the two-tailed t-test. One-way ANOVA was used for comparison of multiple groups. A p-value $\leq 0.05$ was considered statistically significant. Survival curves were plotted using the Kaplan-Meier methods. All statistical analyses were performed by SPSS ver16.0 software.

\section{Results}

Clinical manifestations of aGVHD are alleviated in mice treated with anti-IL-18R $\alpha A b$. To investigate the protective role of IL-18R blockage in aGVHD, BMCs from C57BL/6 mice were transplanted into irradiated BALB/c mice as described in Materials and methods. Recipient mice were then divided into two experimental groups. The $\mathrm{BS}+\mathrm{Ab}$ group received intraperitoneal injection of $10 \mu \mathrm{g}$ neutralizing anti-IL-18R $\alpha$ $\mathrm{mAb}$, while animals in the control BS group were treated with an intraperitoneal injection of PBS. The characteristics of aGVHD, such as mean weight loss, clinical score, and survival rate were assessed in each group. Seven days after transplantation, the mice in both groups exhibited characteristic clinical symptoms of aGVHD, including hunch posture, hair loss and skin lesions (Fig. 1A). All irradiated mice lost weight that peaked on day 14 P.T., with significantly less weight loss observed in the $\mathrm{BS}+\mathrm{Ab}$ group (compared to the BS group (19 \pm 1.5 and $27 \pm 2.4 \%$, respectively, $\mathrm{P}<0.05$, Fig. $1 \mathrm{~B})$. During the entire observation period of 35 days, mice in the $\mathrm{BS}+\mathrm{Ab}$ group had a significantly lower clinical score than the BS group, with the difference reaching its maximum at 14 P.T. $(\mathrm{P}<0.05$, Fig. 1C). We detected a markedly higher survival rate of mice in the $\mathrm{BS}+\mathrm{Ab}$ group compared to the $\mathrm{BS}$ group (Fig. 1D). Additionally, the manifestations of aGVHD in the $\mathrm{BS}+\mathrm{Ab}$ group were milder than those in the BS group until the end of the study (35 days P.T.). Taken together, these results indicated that blocking the interaction of IL-18 with IL-18R by anti-IL-18R $\alpha \mathrm{mAb}$ attenuated the clinical manifestations of aGVHD in the mouse animal model.

Anti-IL-18R $\alpha$ Ab administration decreases systemic inflammation in the aGVHD mouse model. Systemic inflammation is associated with the expression of Th cell subsets (17). We analyzed Th subsets in the peripheral blood of the BS+Ab and BS aGVHD mice at various time-points using FACS analysis. In both groups, the percentage of Th1 cells increased reaching its peak at day 21 P.T. (Fig. 2A). BS+Ab mice exhibited significantly reduced levels of Th1 cells 7 and 14 days P.T. as compared to the $\mathrm{BS}$ group $(\mathrm{P}<0.05)$. This difference was no longer observed at 21-35 days P.T., as the percentage of Th1 cells in both groups gradually decreased to pre-transplantation levels (Fig. 2A). Different dynamic changes were observed in the levels of Th2 and Th17 cells. The BS+Ab group exhibited a markedly lower percentage of Th2 cells in the peripheral blood when compared to the BS group on day 21 and 35 (Fig. 2B, $\mathrm{P}<0.05$ ), while no significant difference was detected at earlier time-points. Levels of Th17 cells rose slowly in both groups during the same period, with the percentage of Th17 cells 
A

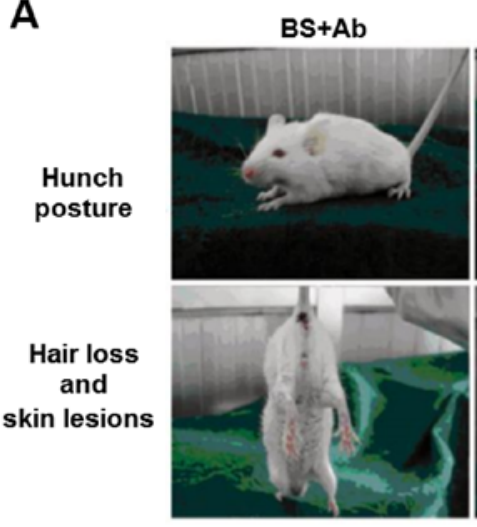

C

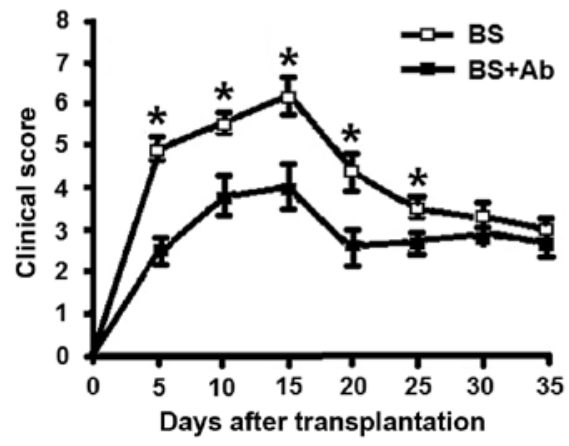

BS

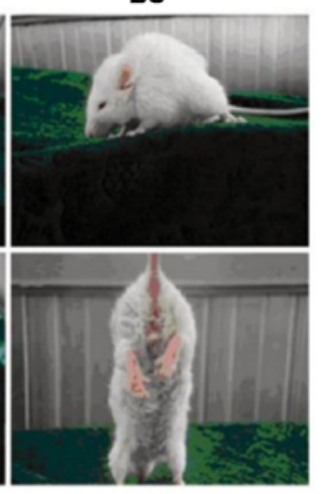

BS

B
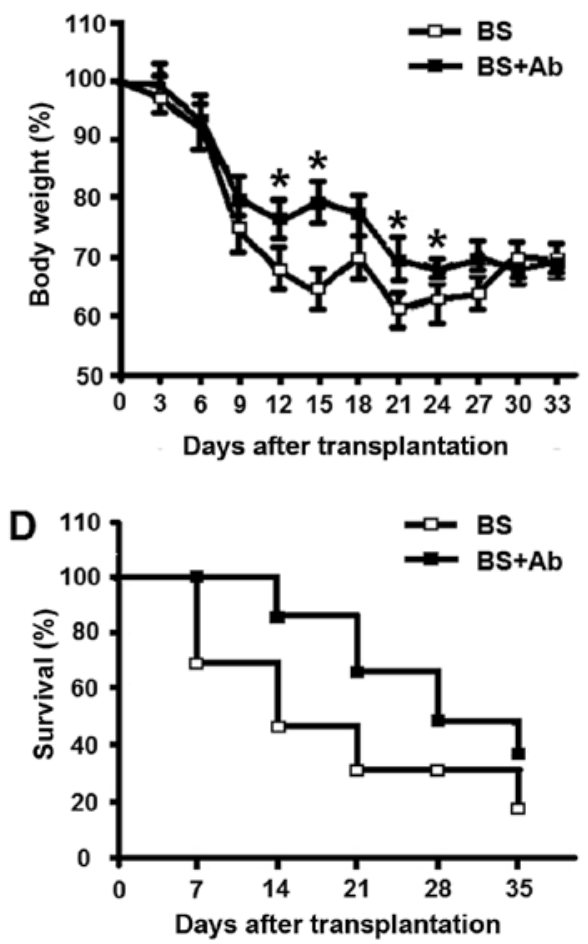

Figure 1. Anti-IL-18R $\alpha$ mAb alleviates aGVHD systemic symptoms in aGVHD mice. aGVHD was induced in irradiated BALB/c mice by BMC transplantation. Ten micrograms of anti-IL-18R $\alpha$ mAb was administered by intraperitoneal injection to mice in the BS+Ab group, while the BS group received injection of PBS. (A) Hunch posture, hair loss and skin lesions were evaluated on day 14 P.T. (B) Changes in body weight of the recipient mice at different time-points in the different groups. (C) aGVHD clinical scores of the BS+Ab and BS groups on day 14 P.T. (D) Survival rates of aGVHD mice in the different groups. $\mathrm{n}=6$ in each group. * $\mathrm{P}<0.05$. GVHD, graft-versus-host disease; IL-18, interleukin-18; aGVHD, acute GVHD; BMC, bone marrow cell; PBS, phosphate-buffered solution; mAb, monoclonal antibody; P.T., post-transplantation.

being lower in the $\mathrm{BS}+\mathrm{Ab}$ group than that in the BS group on day 7 P.T., but higher on days 21 and 35 P.T. (Fig. 2C). Taken together, these results suggest that blocking IL-18R had differential effects on the differentiation of the various Th subsets.

Serum levels of IFN- $\gamma$, IL-17A, IL-4, IL-6 and IL-18 are associated with the inflammatory response (18-20). We next evaluated serum levels of these cytokines in the $\mathrm{BS}+\mathrm{Ab}$ and BS groups of the aGVHD mice at different time-points using cytometric bead array and ELISA as described in Materials and methods. Mice in the $\mathrm{BS}+\mathrm{Ab}$ group exhibited significantly elevated serum levels of IFN- $\gamma$ as compared to the BS group (Fig. 2D). Similarly, serum levels of IL-4 and IL-17A in the BS+Ab group were markedly higher on day 35 P.T. (Fig. 2E and F), while serum IL-18 was elevated in the $\mathrm{BS}+\mathrm{Ab}$ group during the entire period of observation (Fig. 2G). On the other hand, animals in $\mathrm{BS}+\mathrm{Ab}$ group had significantly lower levels of IL-6 in the first 4 weeks P.T., followed by a dramatic increase in serum IL- 6 concentration on day 35 P.T. (Fig. 2H). Taken together, these results suggest that IL-18R blockade mediates the inflammatory response through regulation of cytokine secretion.

The main target organs of aGVHD are the liver and small intestine (21). We next addressed the effect of IL-18R blockage by anti-IL-18R $\alpha$ on inflammation and necrosis in the tissues of the aGVHD mouse model. Histopathological analysis of the liver and small intestine showed that on day 21 P.T. the degree of inflammation and necrosis in the mice of the BS group was more severe than that in the $\mathrm{BS}+\mathrm{Ab}$ and normal control group (untreated animals), as indicated by the intensity of $\mathrm{H} \& \mathrm{E}$ staining (Fig. 2I). These results correlated with a significantly increased histopathologic score detected in both the liver and small intestine of the BS group as compared to the score in the $\mathrm{BS}+\mathrm{Ab}$ group (Fig. $2 \mathrm{~J}, \mathrm{P}<0.05$ ). Taken together, these data imply that the administration of anti-IL-18R $\alpha \mathrm{mAb}$ affects the inflammatory response and the pathological progression of aGVHD in main target organs.

Apoptosis and expression of apoptosis-related protein are reduced by anti-IL-18R $\alpha$ Ab treatment. Numerous studies have shown that the cell apoptosis rate is increased in experimental GVHD mouse models $(22,23)$. In order to assess the influence of anti-IL-18R $\alpha$ mAb on cell apoptosis in aGVHD target organs, we evaluated the percentage of apoptotic cells in the liver and small intestine of aGVHD mice on day 14 P.T. by TUNEL assay. The liver and small intestine of the BS group animals showed increased expression of apoptotic cells as compared to the normal group (untreated animals) (Fig. 3). On the other hand, administration of anti-IL-18R $\alpha \mathrm{mAb}$ in the $\mathrm{BS}+\mathrm{Ab}$ group (experimental group) markedly reduced TUNEL staining of the liver and small intestine, suggesting that blocking IL-18R inhibited aGVHD-induced cell apoptosis.

Next, we addressed the mechanism of the effect of anti-IL-18R $\alpha \mathrm{mAb}$ on cell apoptosis in aGVHD. Since the activity of IL-18 in different organ tissues is determined by the infiltration of IL-18 into these tissues, we analyzed mRNA and protein levels of IL-18 in the liver and small intestine on 

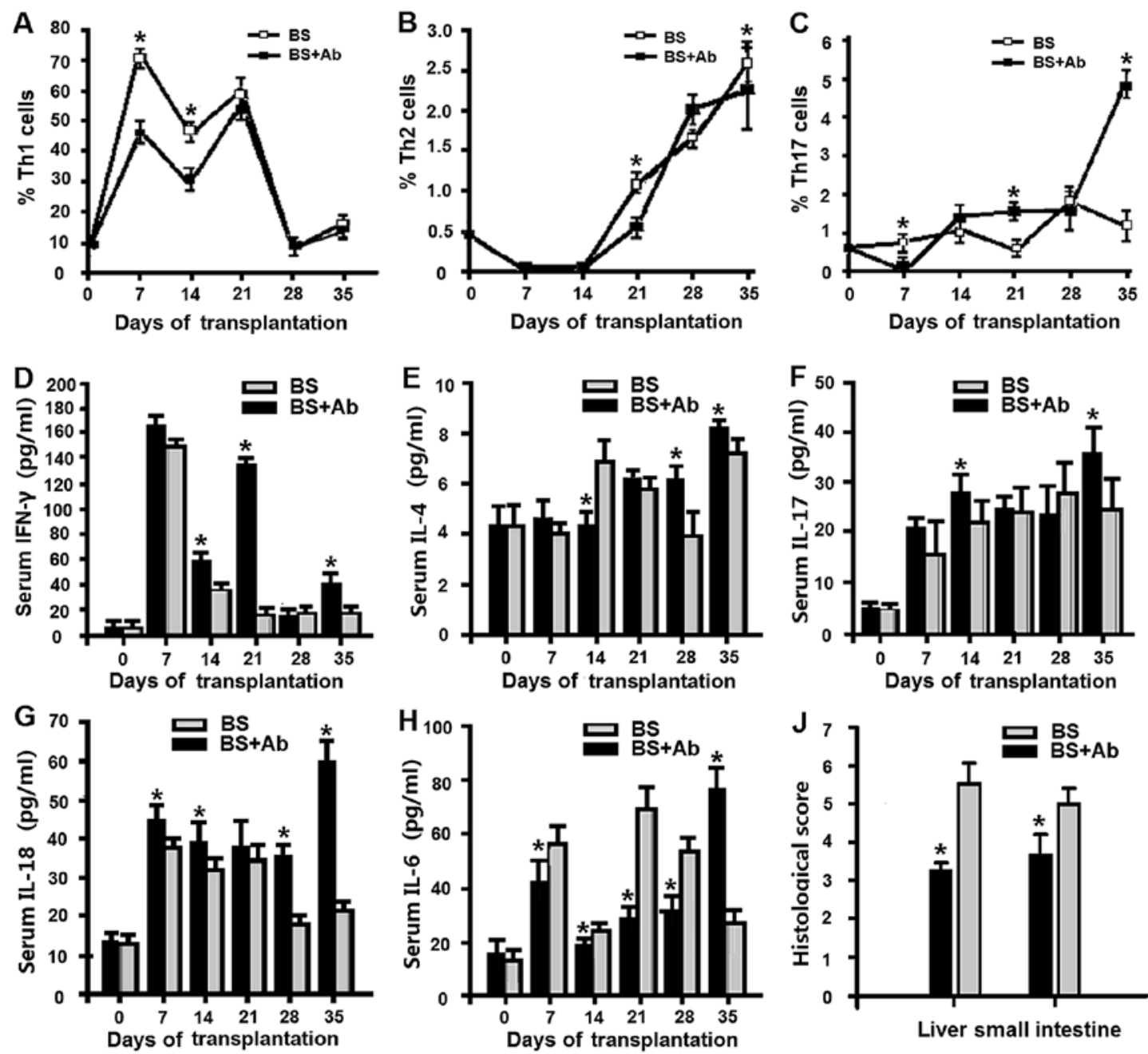

I

Normal

BS
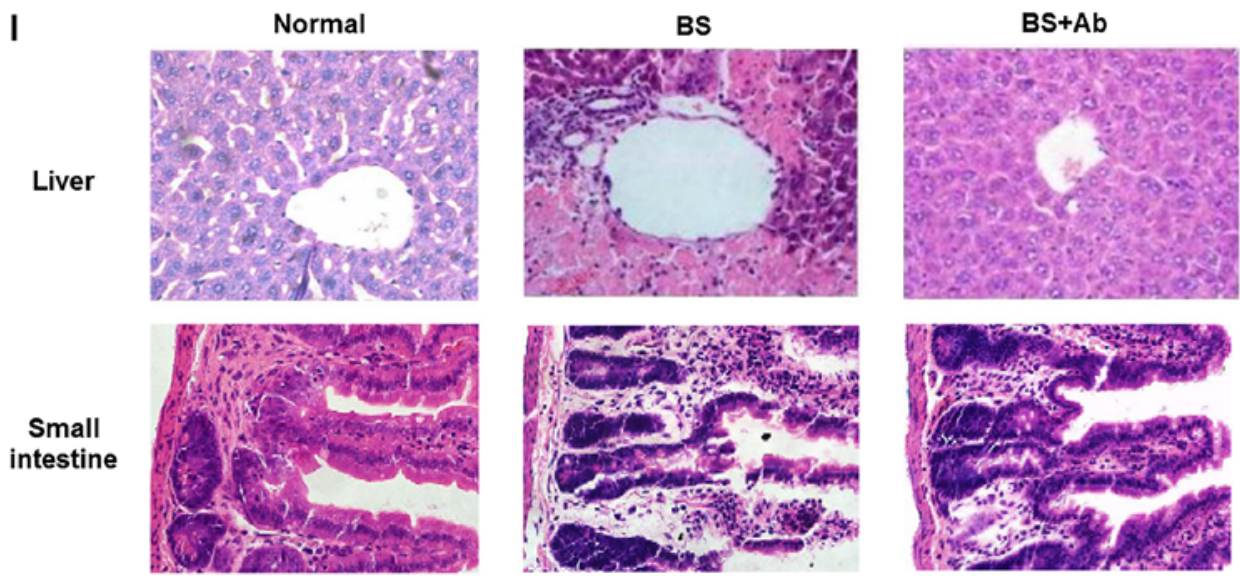

Figure 2. Effect of anti-IL-18R $\alpha \mathrm{mAb}$ administration on Th cell subsets, pro-inflammatory cytokines and histological scores in the aGVHD mice. (A-C) Peripheral blood levels of Th1 (A), Th2 (B) and Th17 (C) cell subsets in the BS+Ab and BS experimental groups were measured by flow cytometry at different time-points. Serum levels of IFN- $\gamma($ D), IL-4 (E), IL-17A (F) and IL-6 (H) at different time-points were detected by cytometric bead array, and IL-18 levels (G) were measured by ELISA. (I) Representative H\&E staining of the liver and small intestine tissues of the mice in the BS+Ab and BS groups and the normal control group (untreated animals) on day 14 P.T. Magnification, x400. (J) Histological score was measured on day 14 P.T. $n=6$ in each group, ${ }^{*} \mathrm{P}<0.05$. GVHD, graft-versus-host disease; aGVHD, acute GVHD; mAb, monoclonal antibody; Th, T helper; IL-18, interleukin-18; ELISA, enzyme-linked immunosorbent assay; H\&E, hematoxylin and eosin; P.T., post-transplantation.

day 14 P.T. using qPCR and histopathological analysis, respectively. The expression of IL-18 mRNA was markedly increased in the BS group when compared to the level in the normal and BS+Ab group (Fig. 4). These results correlated with the increased IL-18 protein expression in the affected tissues of the BS group as compared to the $\mathrm{BS}+\mathrm{Ab}$ and normal control animals (Fig. 5). Increased expression of Fas and FasL serves as a reliable marker of apoptosis and has been implicated in GVHD pathogenesis $(23,24)$. To determine whether these apoptosis-related proteins were affected by anti-IL-18R $\alpha$ treat- 
A

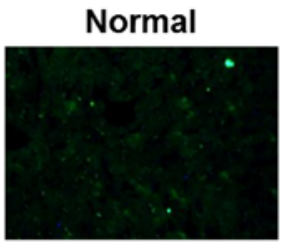

$B S+A b$

BS

Liver
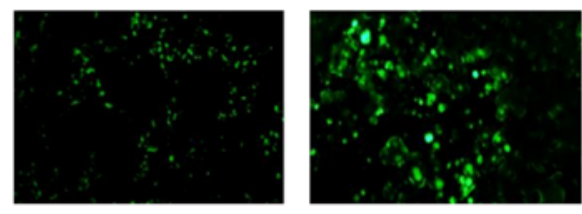

Small intestine
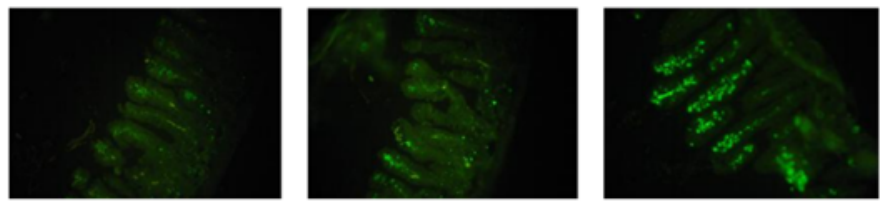

B

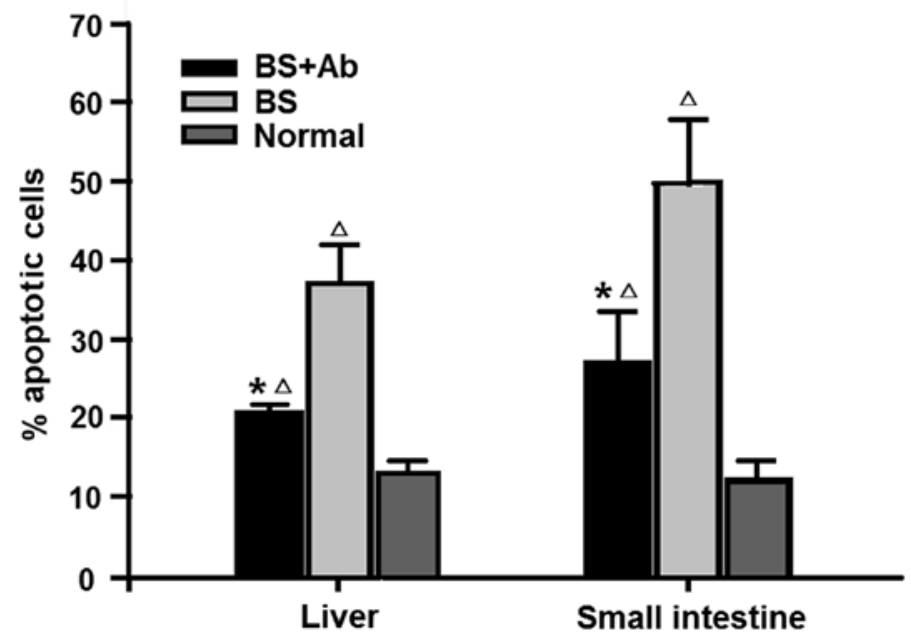

Figure 3. Cell apoptosis in the liver and small intestine of the aGVHD mice on day 14 P.T. (A) Cell apoptosis in the liver and small intestine of the BS+Ab, BS and normal control mice was analyzed using TUNEL assay. (B) Quantitative analysis of the apoptotic cells in the 3 experimental groups. $n=4$ in each group, ${ }^{*} \mathrm{P}<0.05$, compared with the BS group, ${ }^{\triangle} \mathrm{P}<0.05$, compared with the normal group. GVHD, graft-versus-host disease; aGVHD, acute GVHD; P.T., post-transplantation.
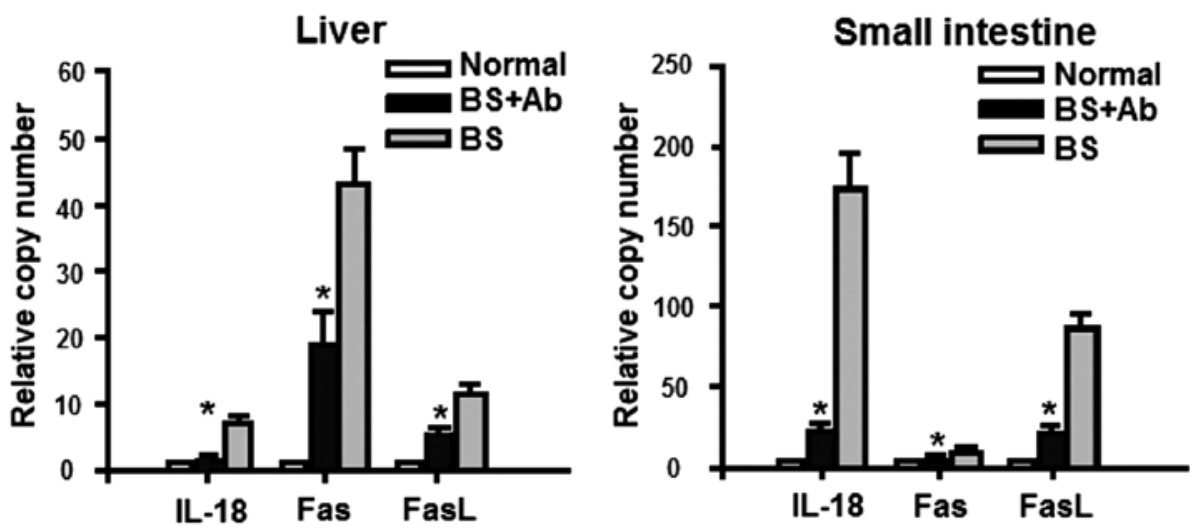

Figure 4. mRNA expression levels of IL-18, Fas and FasL in the liver and small intestine of the aGVHD mice on day 14 P.T. The mRNA levels of IL-18, Fas and FasL in the liver, and small intestine of the BS+Ab, BS and normal control mice were normalized to $\beta$-actin and presented as relative copy number. $\mathrm{n}=4$ in each group. ${ }^{*} \mathrm{P}<0.05$. IL-18, interleukin-18; GVHD, graft-versus-host disease; aGVHD, acute GVHD; P.T., post-transplantation.

ment, we analyzed the expression of Fas and FasL in the liver and small intestine of aGVHD mice on day 14 P.T. We found that the mRNA expression levels of both Fas and FasL were significantly lower in the BS+Ab group than levels in the BS group (Fig. 4, $\mathrm{P}<0.05$ ), and similar to expression levels detected in the untreated control group. Similarly, histopathological analysis of the liver and small intestine tissues of the BS+Ab animals showed decreased protein levels of Fas and FasL as compared to the BS experimental group (Fig. 5). Together these data revealed that administration of IL-18R $\alpha$ mAb may decrease IL-18 infiltration into the liver and small intestine, as well as diminish Fas and FasL levels in these target organs in aGVHD mice.

Anti-IL-18R a Ab treatment reduces apoptosis-related p38MAPK phosphorylation. Previous studies suggest that 


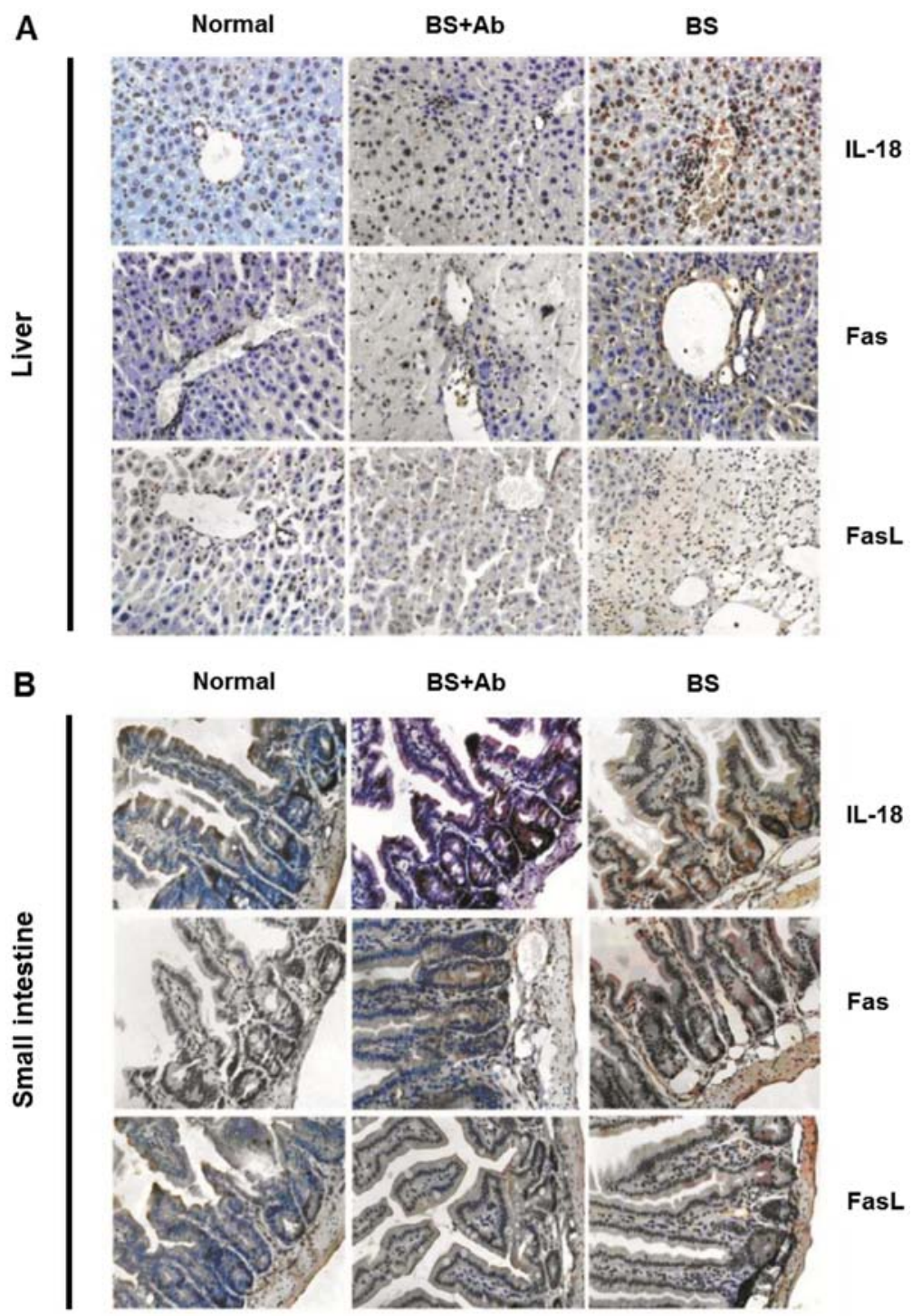

Figure 5. Detection of IL-18, Fas and FasL in the liver and small intestine tissues of the aGVHD mice on day 14 P.T. Paraffin-embedded liver (A) and small intestinal (B) tissues from the BS+Ab, BS and normal control mice were sectioned and analyzed by IHC using IL-18, Fas and FasL antibodies. Magnification, x400. IL-18, interleukin-18; GVHD, graft-versus-host disease; aGVHD, acute GVHD; P.T., post-transplantation; IHC, immunohistochemistry.
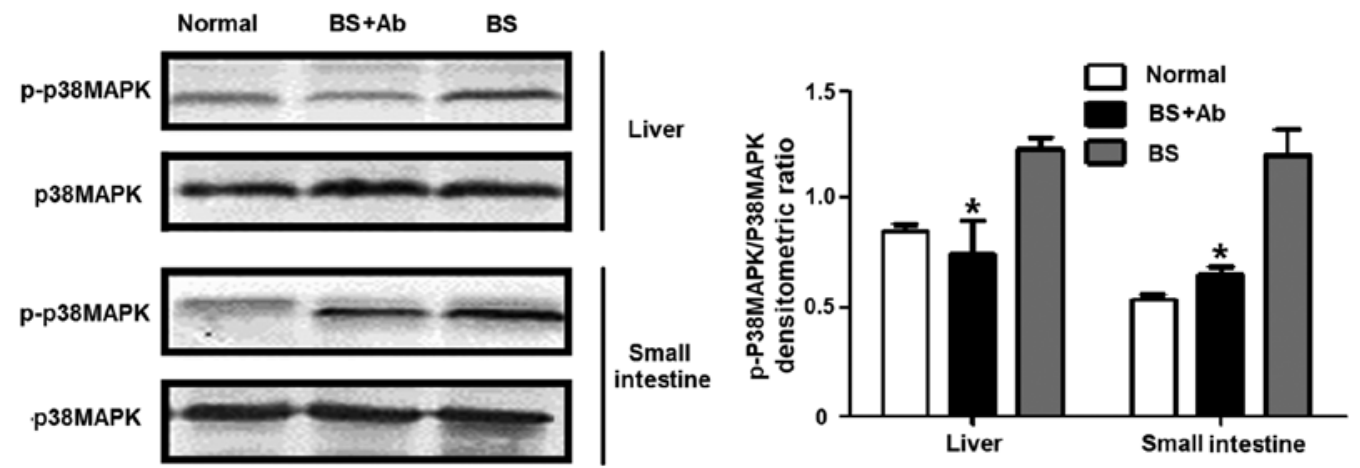

Figure 6. Total p38MAPK and p-p38MAPK expression in the liver and small intestine of the aGVHD mice. Fourteen days post-transplantation, total proteins were extracted from the liver and small intestine of the BS+Ab, BS and normal control mice, and levels of p38MAPK and p-p38MAPK were evaluated by western blotting and densitometry as described in Materials and methods. $n=4$ in each group, $\mathrm{P}<0.05$. GVHD, graft-versus-host disease; aGVHD, acute GVHD; p-p38MAPK, phosphorylated p38MAPK.

the p38MAPK signaling pathway is involved in cell apoptosis mediated by Fas/FasL (25). Since anti-IL-18R $\alpha$ mAb administration clearly diminished Fas and FasL levels in the liver and small intestine of the aGVHD animals, we next analyzed the effect of IL-18 blockage on the levels of p38MAPK activity in the liver and small intestine of the aGVHD animals by 
assessing p38MAPK phosphorylation on day 14 P.T. As shown in Fig. 6, the levels of p38MAPK phosphorylation were markedly increased in the liver and small intestine of the BS group comparing to levels in the normal group on day 14 P.T. $(\mathrm{P}<0.05)$, while IL-18R blockage with anti-IL-18R $\alpha \mathrm{mAb}$ (BS+Ab group) led to a decrease in $\mathrm{p} 38 \mathrm{MAPK}$ phosphorylation to levels comparable to the untreated control animals $(\mathrm{P}<0.05$ compared to the BS group).

Together, these results suggest that IL-18 binding to IL-18R $\alpha$ induces p38MAPK phosphorylation that is correlated with increased Fas/FasL expression in the liver and intestine of aGVHD animals. The suppression of p38MAPK activity and Fas/FasL expression in aGVHD mice by anti-IL-18R $\alpha$ $\mathrm{mAb}$ administration is associated with a decrease in cell apoptosis.

\section{Discussion}

Previous studies suggest an important role of IL-18 in the pathogenesis of numerous conditions, such as lupus nephritis, and rheumatoid arthritis $(26,27)$. IL-18 is overexpressed in patients with aGVHD and in experimental aGVHD animal models, and plays an important role in the progression of the disease $(28,29)$. Previous studies have demonstrated that antiIL-18R $\alpha$ antibody administration could efficiently alleviate lung inflammation and attenuate the development of necrotizing enterocolitis $(30,31)$. In the present study we evaluated the possible protective effect of the anti-IL-18R $\alpha$ antibody on mice with experimental aGVHD. Our results confirmed that administration of the anti-IL-18R $\alpha \mathrm{mAb}$ to irradiated mice after BMC transplantation significantly reduced the severity of aGVHD symptoms, such as hunch posture, hair loss, skin lesions and weight loss. Our results therefore suggest that anti-IL-18R $\alpha$ therapy could potentially abrogate the biological effect of IL-18 on the onset of aGVHD and is beneficial for reducing the clinical symptoms of cGVHD.

aGVHD is often associated with changes in subsets of Th cells. We found that blocking IL-18R markedly affected the levels of Th1, Th2 and Th17 subsets in the peripheral blood of aGVHD animals, but with different dynamics. Anti-IL-18R $\alpha$ antibody administration led to a significant but transient decrease in Th1 levels 7 and 14 days P.T., with Th1 levels decreasing gradually to control levels by day 35 P.T. in all experimental groups. In contrast, the percentage of Th2 cells in mice treated with anti-IL-18R $\alpha$ antibody was similar to the untreated aGVHD animals at early time-points, but decreased significantly on days 21 and 35 P.T., while Th17 levels were initially decreased in the anti-IL-18R $\alpha$ mAb-treated mice as compared to the aGVHD animals. These animals exhibited a marked increase in the percentage of Th17 cells at later time-points (21 and 35 days P.T.). Different dynamics in these changes suggest that in aGVHD Th cell subsets may be mediated by different factors.

In contrast with the decreased levels of Th1 cells in the anti-IL-18R $\alpha$ mAb-treated mice with aGVHD, we found elevated expression of IFN- $\gamma$, a cytokine secreted mainly by Th1 cells, in this experimental group. Although Th1 cells are considered the main source of IFN- $\gamma$, other immune cells also have the potential to secrete this cytokine (32). It is possible that blocking IL-18 may influence the function of these immune cells, leading to increased secretion of IFN- $\gamma$. The exact source of IFN- $\gamma$ in the anti-IL-18R $\alpha$ mAb-treated aGVHD mice merits further investigation.

Administration of anti-IL-18R $\alpha \mathrm{mAb}$ led to significant attenuation of IL-4 and IL-17A serum concentrations as compared to the aGVHD animals, but the dynamics of these changes were different, suggesting that these two subsets of cytokines may also be mediated by different factors in aGVHD. Notably, anti-IL-18R $\alpha$ mAb also induced an increase in the serum level of IL-18 in the aGVHD mice. We may speculate that the anti-IL-18R $\alpha$ antibody may hinder IL-18 from binding to IL-18R, leading to serum IL-18 accumulation. At the same time, anti-IL-18R $\alpha$-treated aGVHD mice exhibited markedly reduced IL-18 expression in the liver and small intestine as compared with the untreated aGVHD animals, suggesting that blocking the interaction of IL-18 with its receptors prevents the accumulation of IL-18 in organ tissues of the aGVHD animals.

Previous studies have shown that IL-18 activates inflammatory cytokines, such as IL-6, to promote the inflammatory response (33). In this study, we found that IL-6 was reduced in the aGVHD mice treated with the anti-IL-18R $\alpha$ antibody during the first 4 weeks P.T., suggesting that blockade of IL-18 is able to inhibit the inflammatory response. Further studies are required to identify the factors associated with the increased expression of IL-6 on day 35 P.T. reported in our study.

The effect of the anti-IL-18R $\alpha$ antibody on pro-inflammatory cytokine levels in aGVHD mice were correlated with decreased inflammation and necrosis detected in the liver and small intestine of animals receiving anti-IL-18R $\alpha$ therapy, suggesting that blocking IL-18R may be beneficial in preventing organ tissue damage.

Studies have shown an association between IL-18 and cell apoptosis in aGVHD (22). We were able to significantly reduce the apoptotic rates in the liver and small intestine of the aGVHD mouse model by interfering with IL-18/IL-18R binding. This effect of IL-18R blockage was associated with the inhibition of Fas and FasL expression. Previous studies suggest that p38MAPK activity mediates Fas/FasL-induced apoptosis (25). Our studies confirm that p38MAPK phosphorylation plays a role in mediating aGVHD, and blocking IL-18R inhibits p38MAPK activation. These results suggest that inhibition of cell apoptosis by the IL-18R $\alpha$ antibody is associated with the downregulation of apoptosis-related proteins and the p38MAPK signaling pathway.

In conclusion, our study indicated that blocking IL-18R with the anti-IL-18R $\alpha$ antibody prevents the pathogenesis in early stages of experimental aGVHD. As an interference method, anti-human IL-18R $\alpha$ may represent a novel strategy for the treatment of human aGVHD. Additional research concerning the utility of IL-18R-based therapeutics to prevent and treat aGVHD is warranted.

\section{Acknowledgements}

This study was supported by the Qinglan Project of Jiangsu Province of China, National Nature Science Foundation of China (grant no. 81070446) and the Priority Academic Program Development of Jiangsu Higher Education Institutions (PAPD). We thank Dr Weidong Du (University Clinic Ulm, Ulm, Germany) for proofreading the manuscript. 


\section{References}

1. Storb R: Allogeneic hematopoietic stem cell transplantation - yesterday, today, and tomorrow. Exp Hematol 31: 1-10, 2003.

2. Ferrara JL, Levine JE, Reddy P and Holler E: Graft-versus-host disease. Lancet 373: 1550-1561, 2009.

3. Tobin LM, Healy ME, English K and Mahon BP: Human mesenchymal stem cells suppress donor CD4(+) T cell proliferation and reduce pathology in a humanized mouse model of acute graft-versus-host disease. Clin Exp Immunol 172: 333-348, 2013

4. Reddy P: Pathophysiology of acute graft-versus-host disease. Hematol Oncol 21: 149-161, 2003.

5. Antin JH and Ferrara JL: Cytokine dysregulation and acute graft-versus-host disease. Blood 80: 2964-2968, 1992.

6. Levine JE, Paczesny S and Sarantopoulos S: Clinical applications for biomarkers of acute and chronic graft-versus-host disease. Biol Blood Marrow Transplant 18 (Suppl 1): S116-S124, 2012.

7. Pan B, Zhang Y, Sun Y, Cheng H, Wu Y, Song G, Chen W, Zeng L and Xu K: Deviated balance between Th1 and Th17 cells exacerbates acute graft-versus-host disease in mice. Cytokine 68 : 69-75, 2014.

8. Tawara I, Maeda Y, Sun Y, Lowler KP, Liu C, Toubai T, McKenzie AN and Reddy P: Combined Th2 cytokine deficiency in donor $\mathrm{T}$ cells aggravates experimental acute graft-vs-host disease. Exp Hematol 36: 988-996, 2008.

9. Reddy P, Arora M, Guimond M and Mackall CL: GVHD: A continuing barrier to the safety of allogeneic transplantation. Biol Blood Marrow Transplant 15 (Suppl 1): 162-168, 2009.

10. Schmaltz C, Alpdogan O, Horndasch KJ, Muriglan SJ, Kappel BJ, Teshima T, Ferrara JL, Burakoff SJ and van den Brink MR: Differential use of Fas ligand and perforin cytotoxic pathways by donor T cells in graft-versus-host disease and graft-versus-leukemia effect. Blood 97: 2886-2895, 2001.

11. Maeda Y, Levy RB, Reddy P, Liu C, Clouthier SG, Teshima T and Ferrara JL: Both perforin and Fas ligand are required for the regulation of alloreactive $\mathrm{CD} 8^{+} \mathrm{T}$ cells during acute graft-versus-host disease. Blood 105: 2023-2027, 2005.

12. Fremond CM, Togbe D, Doz E, Rose S, Vasseur V, Maillet I, Jacobs M, Ryffel B and Quesniaux VF: IL-1 receptor-mediated signal is an essential component of MyD88-dependent innate response to Mycobacterium tuberculosis infection. J Immunol 179: 1178-1189, 2007.

13. Chandrasekar B, Vemula K, Surabhi RM, Li-Weber M, Owen-Schaub LB, Jensen LE and Mummidi S: Activation of intrinsic and extrinsic proapoptotic signaling pathways in interleukin-18-mediated human cardiac endothelial cell death. J Biol Chem 279: 20221-20233, 2004

14. Lu Y, Sakamaki S, Kuroda H, Kusakabe T, Konuma Y, Akiyama T, Fujimi A, Takemoto N, Nishiie K, Matsunaga T, et al: Prevention of lethal acute graft-versus-host disease in mice by oral administration of T helper 1 inhibitor, TAK-603. Blood 97: 1123-1130, 2001.

15. Cooke KR, Kobzik L, Martin TR, Brewer J, Delmonte J Jr, Crawford JM and Ferrara JL: An experimental model of idiopathic pneumonia syndrome after bone marrow transplantation: I. The roles of minor $\mathrm{H}$ antigens and endotoxin. Blood 88 : 3230-3239, 1996.

16. Ivanov II, McKenzie BS, Zhou L, Tadokoro CE, Lepelley A, Lafaille JJ, Cua DJ and Littman DR: The orphan nuclear receptor RORgammat directs the differentiation program of proinflammatory IL-17 ${ }^{+} \mathrm{T}$ helper cells. Cell 126: 1121-1133, 2006.

17. Sun Y, Tawara I, Toubai T and Reddy P: Pathophysiology of acute graft-versus-host disease: Recent advances. Transl Res 150: $197-214,2007$.
18. Wilson SP and Cassel SL: Inflammasome-mediated autoinflammatory disorders. Postgrad Med 122: 125-133, 2010.

19. Miossec P: IL-17 and Th17 cells in human inflammatory diseases. Microbes Infect 11: 625-630, 2009.

20. Nishimoto $\mathrm{N}$ and Kishimoto T: Interleukin 6: From bench to bedside. Nat Clin Pract Rheumatol 2: 619-626, 2006.

21. Vogelsang GB, Lee L and Bensen-Kennedy DM: Pathogenesis and treatment of graft-versus-host disease after bone marrow transplant. Annu Rev Med 54: 29-52, 2003.

22. Reddy P, Teshima T, Kukuruga M, Ordemann R, Liu C, Lowler K and Ferrara JL: Interleukin-18 regulates acute graft-versus-host disease by enhancing Fas-mediated donor T cell apoptosis. J Exp Med 194: 1433-1440, 2001.

23. Wasem C, Frutschi C, Arnold D, Vallan C, Lin T, Green DR Mueller $\mathrm{C}$ and Brunner T: Accumulation and activation-induced release of preformed Fas (CD95) ligand during the pathogenesis of experimental graft-versus-host disease. J Immunol 167: 2936-2941, 2001.

24. Yi T, Zhao D, Lin CL, Zhang C, Chen Y, Todorov I, LeBon T, Kandeel F, Forman S and Zeng D: Absence of donor Th17 leads to augmented Th1 differentiation and exacerbated acute graft-versus-host disease. Blood 112: 2101-2110, 2008.

25. Kalina U, Kauschat D, Koyama N, Nuernberger H, Ballas K, Koschmieder S, Bug G, Hofmann WK, Hoelzer D and Ottmann OG: IL-18 activates STAT3 in the natural killer cell line 92, augments cytotoxic activity, and mediates IFN-gamma production by the stress kinase p38 and by the extracellular regulated kinases p44 ${ }^{\text {erk-1 }}$ and p42 ${ }^{\text {erk-21 }}$. J Immunol 165: 1307-1313, 2000.

26. Calvani N, Tucci M, Richards HB, Tartaglia P and Silvestris F: Th1 cytokines in the pathogenesis of lupus nephritis: The role of IL-18. Autoimmun Rev 4: 542-548, 2005.

27. Gracie JA, Forsey RJ, Chan WL, Gilmour A, Leung BP, Greer MR, Kennedy K, Carter R, Wei XQ, Xu D, et al: A proinflammatory role for IL-18 in rheumatoid arthritis. J Clin Invest 104: 1393-1401, 1999.

28. Miller WP, Srinivasan S, Panoskaltsis-Mortari A, Singh K, Sen S, Hamby K, Deane T, Stempora L, Beus J, Turner A, et al: GVHD after haploidentical transplantation: A novel, MHC-defined rhesus macaque model identifies $\mathrm{CD} 28^{-} \mathrm{CD}^{+} \mathrm{T}$ cells as a reservoir of breakthrough T-cell proliferation during costimulation blockade and sirolimus-based immunosuppression. Blood 116: 5403-5418, 2010.

29. Arnold D, Wasem C, Juillard P, Graber P, Cima I, Frutschi C, Herren S, Jakob S, Alouani S, Mueller C, et al: IL-18-independent cytotoxic T lymphocyte activation and IFN-gamma production during experimental acute graft-versus-host disease. Int Immunol 14: 503-511, 2002.

30. Kang MJ, Homer RJ, Gallo A, Lee CG, Crothers KA, Cho SJ, Rochester C, Cain H, Chupp G, Yoon HJ, et al: IL-18 is induced and IL-18 receptor alpha plays a critical role in the pathogenesis of cigarette smoke-induced pulmonary emphysema and inflammation. J Immunol 178: 1948-1959, 2007.

31. Halpern MD, Khailova L, Molla-Hosseini D, Arganbright K, Reynolds C, Yajima M, Hoshiba J and Dvorak B: Decreased development of necrotizing enterocolitis in IL-18-deficient mice. Am J Physiol Gastrointest Liver Physiol 294: G20-G26, 2008.

32. Schroder K, Hertzog PJ, Ravasi T and Hume DA: Interferon-gamma: An overview of signals, mechanisms and functions. J Leukoc Biol 75: 163-189, 2004.

33. Olee T, Hashimoto S, Quach J and Lotz M: IL-18 is produced by articular chondrocytes and induces proinflammatory and catabolic responses. J Immunol 162: 1096-1100, 1999. 